\title{
Transferring Design Knowledge: Challenges and Opportunities
}

\author{
Jun Hu, Wei Chen, Christoph Bartneck, and Matthias Rauterberg \\ Designed Intelligence Group, Department of Industrial Design \\ Eindhoven University of Technology \\ Den Dolech 2, 5612AZ, Eindhoven, The Netherlands
}

\begin{abstract}
Design becomes more and more the art of bringing together expertise and experts from different domains in creating future products. Synthetical knowledge and hands-on skills in design, especially in industrial design, is often implicit, hardly captured and modeled for remote education. The need of transferring implicit design knowledge using computer mediated learning tools, provides not only technical challenges, but also many research opportunities. In this article the literature about training transfer and implicit design knowledge transfer is reviewed. A scenario of using such learning tools for learning and teaching physical modeling in industrial design is presented, followed by a discussion about the challenges and opportunities in developing such a system.
\end{abstract}

\section{Introduction}

Design becomes more and more the art of bringing together expertise and experts from different domains in creating future products. For example, knowledge from the fields of engineering, ergonomics and psychology are needed for designing future aircraft seat [20]; while designing the next generation of health monitoring systems requires the integration of expertise from medical science, electrical engineering and user research [76]. This multi-disciplinary character requires changes of the design environments, methods and tools in all phases of the development cycle. Besides the analysis based approach, effective and adequate design synthesis is required. In this, synthesis implies composing design elements and approaches, so as to form a whole, as well as combining diverse conceptions into a coherent whole. Design synthesis therefore relies on e.g. adequate process descriptions and methods, on structured means for information and knowledge management, on effective (analysis and simulation) tools, on stimulating (virtual reality) environments and on suitable methods to govern the entire process. But the decisive factor is the effective and far-reaching facilitation of the interaction between designers, domain experts and users.

Most of the computer-aided learning systems are focused on the analytical knowledge and skills. Synthetical knowledge and hands-on skills in design, especially in industrial design, are essential for designers, which requires the education and the learning process to adapt to this need [14/22 13/1]. These knowledge and skills are often implicit, hardly captured and modeled for remote education. For example, as key steps in industrial design, physical models are created to 
visualize the ideas and concepts, to engage the end users and the domain experts for their feedback (for example in [18]). Skills in creating these models are essential, but difficult to be learned from text books. They are learned in practise by close observation and through direct hand-to-hand training. Such a process is expensive and not scalable with the number of students and their geographic distribution. From the pedagogical point of view, modeling is very complex as it involves a number of dimensions. Considering the Bloom's taxonomy [4, it involves all the three domains: cognitive, affective and psychomotor.

In this article we first review the literature about training transfer, especially about implicit design knowledge transfer. We then describe a scenario in which implicit design knowledge would be transferred over a computer mediated system. From this scenario requirements for such a system are identified, followed by a discussion about the challenges and opportunities in developing such a system.

\section{Training Transfer and Virtual Training}

Because organizations consider human capital one of the most salient organizational assets in establishing and maintaining a competitive advantage, many are investing considerable resources to support employee and organizational development activities such as training 319. Globalization, technological advancements, and talent wars in recruiting and retaining high performers are among the other major reasons that organizations seek to leverage training outcomes to foster workplace performance improvement, facilitate development of individual and organizational effectiveness, and establish and maintain market share within the rapidly changing business environment [516].

To identify how training interventions influence performance outcomes in organizations, many researchers have conducted training transfer studies, which have revealed numerous findings about the effect of such transfer factors on employee and organizational performance outcomes [12]. Traditional training transfer studies have assessed the separate influence of trainee characteristics, training design, and work climate variables on training transfer in attempting to validate the influence of each of these independent variables on training transfer 2812; few have used integrated approaches and examined the empirical assessment of cross-relationships and influence of those diverse variables in trainees' characteristics, work and job experiences, tool support, and organizational climate on learning and transfer outcomes [21].

The need to identify the mediating mechanisms to link contextual features influencing training transfer has been a pressing research interest among several researchers [15]. For practitioners, identifying good research findings about the integrative and interactive features among different variables in trainees, training design, tool support, and organizational climate can be valuable in improving the effectiveness of delivered training.

Among research in education tools done to date, several approaches such as CAI, ICAI, Micro-world, ITS, ILE, and CSCL have been proposed and many systems have been built within each approach. Innovative technologies such as 
hyper-media, virtual reality, internet, WWW have significantly affected the research community in general. Recent advances are offering an increasing number of innovative and promising learning environments including three-dimensional and two-dimensional virtual worlds as well as computer simulations. These environments differ a lot as to both their technological sophistication and to the types of skills taught, varying for example from immersive 3D environments with haptic feedback of high-fidelity to simulations of complex relational situations, for the learning of "soft design skills" of growing strategic interest to enterprises such as leadership, customer service, coaching, selling etc.

The learning potential of virtual training relies on the possibility for trainees to make a number of significant first-person experiences and to fail in a safe and protected environment. In order to be effective, the training experience should seem real and engaging to trainees, as 'if they are in there' they should feel (psychomotor, emotionally and cognitively) present in the design situation.

It is therefore important to investigate the relationships existing among the factors that are crucial to the transfer of implicit design knowledge in virtual training environments. There is a need to develop a sophisticated tool support in virtual learning environments, trying to define on the one hand the key factors conveying it in training contexts and on the other hand how the tool support contributes to enhance learning efficacy and to support transfer of design knowledge and skills, which is depicted in the following scenario.

\section{Scenario}

This scenario is based on the teaching and learning practice in cardboard modeling. Typical activities for a student to master such a technique are [10]: acquire basic skills in constructing a set of basic forms and mechanisms providing action possibilities; learn to capture the essence of the complex form of existing products, plan the mock-up and construct it accordingly; learn to use modeling for exploring the solution space of a design problem.

Joe is an expert in modeling. He teaches cardboard modeling at a design school in Eindhoven. He usually provides the course every semester for a small group of maximum 10 students because of the hands-on nature of the course, but now he has to provide the course for up to 100 students and he is also invited to give the course for another design school in Hong Kong. Figure 1 and 2 are examples of cardboard models made for exploring rich interaction possibilities.

Joe decides to use the Virtual Trainer (VT) toolkit to face this new didactic challenge. He prepares his course by first making several cardboard models, from simple ones to complex ones. The VT tools record his actions and behaviors in the process of the modeling. These actions are recorded along a timeline in a multimedia digital format. The format integrates an audiovisual stream and semantically annotated data that describe the teacher activity and important properties (i.e. the status) of the physical model as they evolving during the artefact development time. The multimedia material is prepared automatically by recording, analyzing, filtering and composing data. 


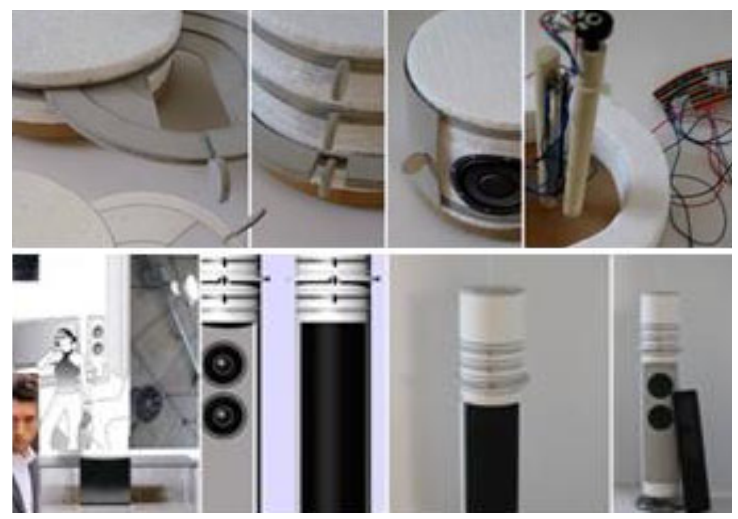

Fig. 1. Cardboard model of a redesigned CD player [11]

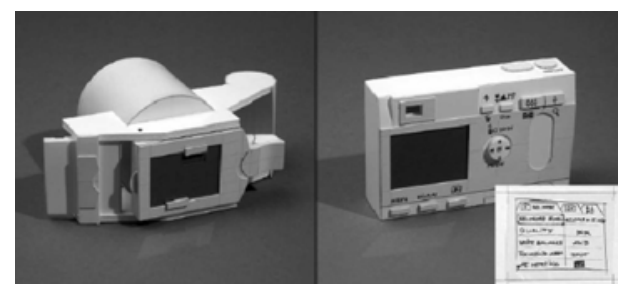

Fig. 2. Cardboard models of cameras designed for rich interaction [9]

When finished with the models, Joe reviews the material and edits some additional personal instruction. Then he packs all the prepared digital material and make it available to his students in Eindhoven and Hong Kong. He asks all his students to follow the online instructions to practise by themselves.

Jackie is one of Joe's students in Hong Kong. Following the online instructions, his assignment today is to make a cardboard model of a camera (Figure 2). He prepares his digitally enhanced tools (the VT toolkit) by loading the digital materials received from Joe. The wearable tools allow Jack to see the cardboards augmented with the cut lines indicating steps, tools and actions. When cutting the cardboards, the tool gives immediate feedback on his actions, on whether a right tool is used, whether it is cut in a right direction, with the right force etc. Suggestions are provided if corrections/improvements are needed.

The intermediate and finalresults are compared to the $3 D$ model and analyzed immediately; feedback and explanations are given if necessary. Multimodal feedback is provided in textual, visual and haptic ways, in order to involve the user in the most suited way.

In this scenario, a learning appliance that supports teaching and learning in $3 \mathrm{D}$ industrial design modeling plays the central role. The appliance consists of a tool that records and annotates a teacher's actions and more importantly, his behaviors, in making a cardboard model, then instructs the students to create the model in a similar manner. 
The appliance consists also of a complementary set of tools, for example wearable devices to be used by students, which allow them to get realtime multimodal feedback about the work they are doing. The feedback is based on the comparison between their model and the way the actions they are taking with the model and the actions of the teacher (see Figure 3 and 4 where the traditional and pro-

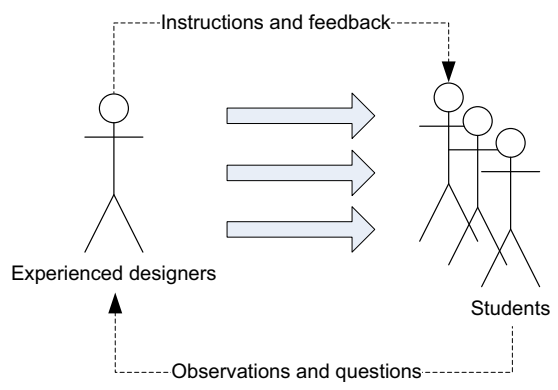

Fig. 3. Traditional learning model posed training models models are depicted). The tools also support communication and remote presence, fostering cooperation among students and strengthening interaction (remote and local) with the teacher. One must notice that the Model in Figure 4 is crucial for capturing the actions and behaviors of the teacher, being transferred over distance, and being utilized for training the students in either real time, or for a later time.

\section{Challenges and Opportunities}

From a technological point of view, the VT learning model involves a number of challenges in order to meet the ambitious objectives. These challenges can be tackled with state of the art technologies provided that the system design is well directed. Enabling technologies include:

- Sensors to detect and characterize the users' (teachers and students) actions and detailed features of the models as they are being prepared. These include for example cameras, laser scanners and wearable sensors.

- Sensor fusion to coordinate data from various sources and automatic generation of 3D models from the sensors.

- Accurate modeling of the teaching and learning activities (considering the three Bloom domains: cognitive, affective and psychomotor) and of the produced/under production artefacts

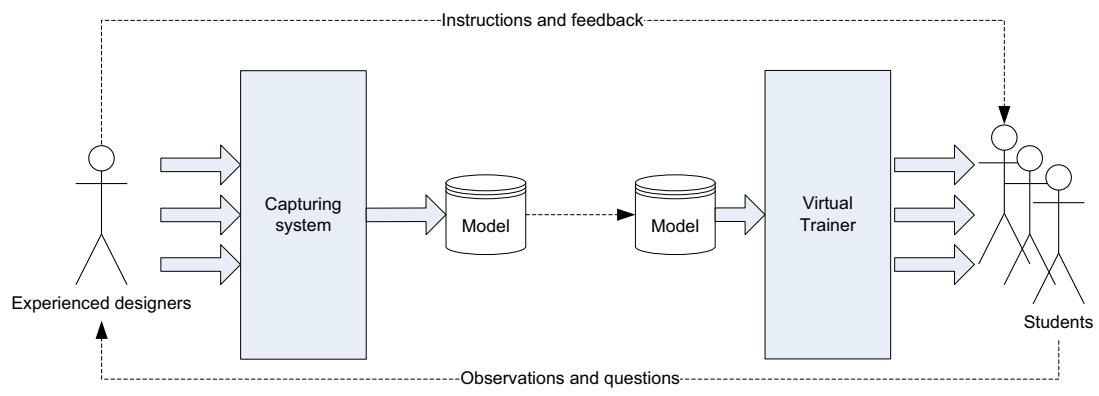

Fig. 4. Proposed learning model 
- Automatic annotation of the human design activities in the digital multimedia stream (according to the user cognitive modeling), for example using ontological engineering approach to externalize the features of the empiricallyformed wisdom or skills of a designer digitally.

- Artificial Intelligence and Machine Learning algorithms in order to train the tools so that they are able to evaluate student activities and their products as compared to teacher's references (also providing feedback for correction and improvement), based on the user cognitive model.

- Human-Computer Interaction in order to make the tools as useful and usable (effective, efficient, pleasant to use) as possible. User interaction modalities also include augmented reality wearable devices and haptic feedback.

From the pedagogical and psychological points of view, such a system can enhance learning processes and skills acquisition, which brings many opportunities to the education and the industry:

- Since the tool can overcome the single tutor-trainee interaction and can be used by many students, whenever and wherever they want, it gives a great training opportunity due to the repeatability of the task; this allows learning to be faster and more effective, since acquiring skills about such complex procedural tasks is grounded on students' management of their learning time and dynamics.

- The system is equipped with teaching support systems and hints implemented according to the guidelines provided by subject matter experts in order to balance reflexive and experiential thinking in doing the task.

- It improves continuous feedback between the student and the virtual tutor, so that each step in learning acquisition can be checked and be part of a whole coherent mental model.

- The interface is designed according to cognitive ergonomics principles of learning transfer, usability and ecological design, to allow students to live a rich and stimulating experience, which will set the stage for basic skills acquisition, and as a further step, a proper ground for creativity development.

- It helps to establish valuable archive/database for interactive teaching and learning, for example, providing model samples and common error examples. The database can be used for evaluating the effectiveness of the teaching and learning process.

It is interesting to utilize an ontological engineering approach analyzing designers' modeling explanation style based on the observable psychomotor design activities. In an activity based ontological engineering approach, defining knowledge as ontology is effective in extracting its essential qualities. it is then interesting to investigate the effectiveness of this new ontological engineering approach (see also [17]), resulting in an ontological framework of designers' explanations: "clarification of the essence of the design style", "discovery of problems in explanations", and "analyzing difficulties in acquiring design style for trainees".

The system also provides the opportunity to investigate the relationships existing among the factors that are crucial to the transfer of implicit design knowledge 
in virtual training environments. In reviewing the training transfer literature, we found that some transfer studies had occurred in international settings in countries in Africa, Europe, and Asia. However, in our review, we seldom found evidence suggesting that studies have examined the empirical assessment of cross-relationships and the influence of those variables. Therefore, developing such a system is unique in that it identifies the cross-relationships and effect of the multi-domain transfer variables on training transfer, and addresses training transfer issues from an international context. The results will prove valuable in expanding the scope of existing research studies of training transfer at the international level based on advanced tool support.

\section{Conclusion}

For transferring implicit design knowledge using computer mediated learning tools, such as the Virtual Trainer system described in this article, requirements are identified and challenges and opportunities are discussed. Among other challenges, how to model of the implicit design knowledge is essential. It is also important to identify the effect of transfer design knowledge according to the relative importance of this knowledge in their influence on trainees' learning, perceived learning applicability, and perceived learning application. Verification of transfer results from post-transfer data such as design performance data will be an important consideration of validation studies. Through these studies, it is possible to reveal meaningful findings in cross-construct settings if other variables in motivation, organizational reward systems, and work ethics from different organizational and cultural settings are used. It is also possible to examine the multi- or uni-dimensional characteristics of design trainer variables as they influence the training transfer processes, and identify when and why certain design trainer variables become converged or distinct under what transfer situation. The need of the virtual training tools for transferring implicit design knowledge provides not only technical challenges, but also many research opportunities.

\section{References}

1. Alers, S., Hu, J.: Admoveo: A robotic platform for teaching creative programming to designers. In: Chang, M., Kuo, R., Kinshuk, Chen, G.-D., Hirose, M. (eds.) Learning by Playing. Game-based Education System Design and Development. LNCS, vol. 5670, pp. 410-421. Springer, Heidelberg (2009)

2. Baldwin, T.T., Ford, J.K.: Transfer of training: A review and directions for future research. The training and development sourcebook, 180 (1994)

3. Becker, B.E., Huselid, M.A., Ulrich, D.: The HR scorecard: Linking people, strategy, and performance. Harvard Business School Pr., Boston (2001)

4. Bloom, B.S.: Taxonomy of educational objectives. Handbook I: Cognitive Domain (1956)

5. Branham, L.: The 7 hidden reasons employees leave. American Management, New York (2005) 
6. Chen, W., Bouwstra, S., Oetomo, S.B., Feijs, L.M.G.: Intelligent Design for Neonatal Monitoring with Wearable Sensors, pp. 386-410. INTECH (2010)

7. Chen, W., Hu, J., Bouwstra, S., Oetomo, S.B., Feijs, L.M.G.: Sensor integration for parinotology. Accepted by International Journal of Sensor Networks IJSNet Special Issue on Recent Advances in Sensor Integration (2010)

8. Ford, J.K., Weissbein, D.A.: Transfer of training: An updated review and analysis. Performance Improvement Quarterly 10, 22-41 (1997)

9. Frens, J.: A rich user interface for a digital camera. Personal and Ubiquitous Computing 10(2), 177-180 (2006)

10. Frens, J.: Dg101 - cardboard modelling, assignment description (2009), https://venus.tue.nl/owinfo-cgi/owi_0695.opl?vakcode=dg101

11. Hendriks, B., Hu, J.: Redesigning a cd player for intuitive rich interaction. In: 12th International Conference on Human-Computer Interaction, CD Proceedings, pp. 1607-1611. Springer, Heidelberg (2007)

12. Holton, E., Bates, R., Ruona, W., Leimbach, M.: Development and validation of a generalized learning transfer climate questionnaire: Final report. In: Proceedings of the 1998 Academy of Human Resource Development Annual Conference, Academy of Human Resource Development, Chicago, IL, pp. 482-489 (1998)

13. Hu, J., Alers, S.: Admoveo: An educational robotic platform for learning behavior programming. In: DeSForM 2009: Design and Semantics of Form and Movement, Taipei, Taiwan, pp. 218-219 (2009)

14. Hu, J., Ross, P., Feijs, L., Qian, Y.: Uml in action: Integrating formal methods in industrial design education. In: Hui, K.-c., Pan, Z., Chung, R.C.-k., Wang, C.C.L., Jin, X., Göbel, S., Li, E.C.-L. (eds.) EDUTAINMENT 2007. LNCS, vol. 4469, pp. 489-498. Springer, Heidelberg (2007)

15. Kozlowski, S.W.J., Farr, J.L.: An integrative model of updating and performance. Human Performance 1(1), 5-29 (1988)

16. Michaels, E., Handfield-Jones, H., Axelrod, B.: The war for talent. Harvard Business School Pr., Boston (2001)

17. Mizoguchi, R., Bourdeau, J.: Using ontological engineering to overcome common ai-ed problems. International Journal of Artificial Intelligence in Education 11(2), 107-121 (2000)

18. van de Mortel, D., Hu, J.: Apartgame: a multiuser tabletop game platform for intensive public use. In: Tangible Play Workshop, Intelligent User Interfaces Conference, Honolulu, Hawaii, USA, pp. 49-52 (2007)

19. Pfeffer, J.: Competitive advantage through people: Unleashing the power of the work force. Harvard Business School Pr., Boston (1994)

20. Tan, C., Chen, W., Kimman, F., Rauterberg, M.: Sleeping posture analysis of economy class aircraft seat. In: Accepted to World Congress on Engineering 2009, London, U.K (2009)

21. Tracey, J.B., Hinkin, T.R., Tannenbaum, S., Mathieu, J.E.: The influence of individual characteristics and the work environment on varying levels of training outcomes. Human Resource Development Quarterly 12(1), 5 (2001)

22. van der Vlist, B., van de Westelaken, R., Bartneck, C., Hu, J., Ahn, R., Barakova, E., Delbressine, F., Feijs, L.: Teaching machine learning to design students. In: Pan, Z., Zhang, X., El Rhalibi, A., Woo, W., Li, Y. (eds.) Edutainment 2008. LNCS, vol. 5093, pp. 206-217. Springer, Heidelberg (2008) 